Georgian Mathematical Journal

Volume 13 (2006), Number 4, 741-749

\title{
COMPLETING THE OPERADIC BUTTERFLY
}

\author{
JEAN-LOUIS LODAY
}

\begin{abstract}
We complete a certain diagram (the operadic butterfly) of the categories of algebras involving Com, As, and Lie by constructing a type of algebras which have 4 generating operations and 16 relations. The associated operad is self-dual for Koszul duality.
\end{abstract}

2000 Mathematics Subject Classification: 18D50, 17Dxx, 17A32.

Key words and phrases: Non-associative algebra, operad, Koszul duality, Zinbiel algebra, Leibniz algebra.

\section{INTRODUCTION}

The three categories of algebras Com, As, and Lie (for commutative algebras, associative algebras and Lie algebras) are related by two functors

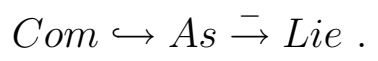

There are other types of algebras with similar functors which make up the following "operadic butterfly":

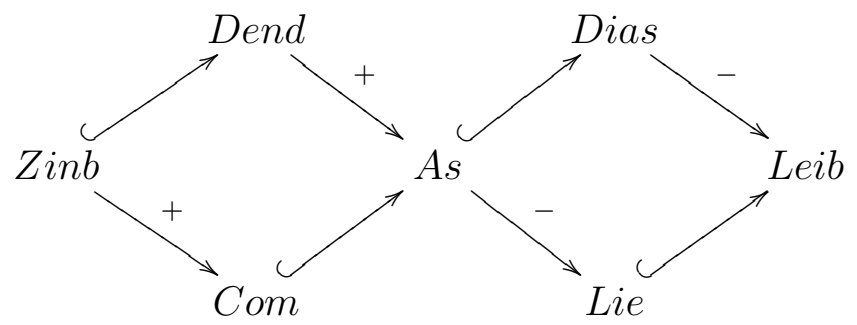

The four other categories of algebras which appear in this diagram are as follows:

Zinb $=$ category of Zinbiel algebras. They have one operation $x \cdot y$ (with no symmetry) satisfying

$$
(x \cdot y) \cdot z=x \cdot(y \cdot z)+x \cdot(z \cdot y) .
$$

Dend $=$ category of dendriform algebras. They have two operations $x \prec y$ and $x \succ y$ (with no symmetry) satisfying

$$
\left\{\begin{aligned}
(x \prec y) \prec z & =x \prec(y \prec z)+x \prec(y \succ z), \\
(x \succ y) \prec z & =x \succ(y \prec z), \\
(x \prec y) \succ z+(x \succ y) \succ z & =x \succ(y \succ z) .
\end{aligned}\right.
$$


Dias $=$ category of diassociative algebras (or associative dialgebras). They have two operations $x \dashv y$ and $x \vdash y$ (with no symmetry) satisfying

$$
\left\{\begin{array}{c}
x \dashv(y \dashv z)=(x \dashv y) \dashv z=x \dashv(y \vdash z), \\
(x \vdash y) \dashv z=x \vdash(y \dashv z), \\
(x \dashv y) \vdash z=x \vdash(y \vdash z)=(x \vdash y) \vdash z .
\end{array}\right.
$$

Leib $=$ category of Leibniz algebras. They have one operation $[x, y]$ (with no symmetry) satisfying

$$
[[x, y], z]=[[x, z], y]+[x,[y, z]] .
$$

An arrow like $\mathcal{A} \hookrightarrow \mathcal{B}$ means that an $\mathcal{A}$-algebra is a $\mathcal{B}$-algebra satisfying some symmetry property. An arrow like $\mathcal{A} \stackrel{ \pm}{\rightarrow} \mathcal{B}$ means that any generating operation of a $\mathcal{B}$-algebra is obtained by some addition (or subtraction) of two operations of the $\mathcal{A}$-algebra type (like the Lie bracket from the associative product). We let the reader to write the exact formulas in each case.

Each one of these types of algebras defines a binary quadratic operad. For these operads there is a well-defined notion of Koszul duality theory devised by Ginzburg and Kapranov [3]. Let $\mathcal{P}^{!}$be the dual of the operad $\mathcal{P}$ (note that $\mathcal{P}^{! !}=\mathcal{P}$ ). It turns out that Koszul duality in the operadic butterfly corresponds to symmetry around the vertical axis passing through $A s$ :

$$
A s^{!}=\text {As, Com }{ }^{!}=\text {Lie, Zinb }{ }^{!}=\text {Leib, Dend }{ }^{!}=\text {Dias . }
$$

A functor of the form $\hookrightarrow$ is changed into a functor of the form $\stackrel{ \pm}{\longleftarrow}$ by duality.

One can slightly enhance the operadic butterfly by putting the category $V e c t$ of algebras with no operation (also called abelian Lie algebras) in between Com and Lie. The operad Vect is self-dual. So an immediate question comes to mind: can one complete the operadic butterfly by putting some category of algebras $\mathcal{X}$ at the upper place on the middle axis ?

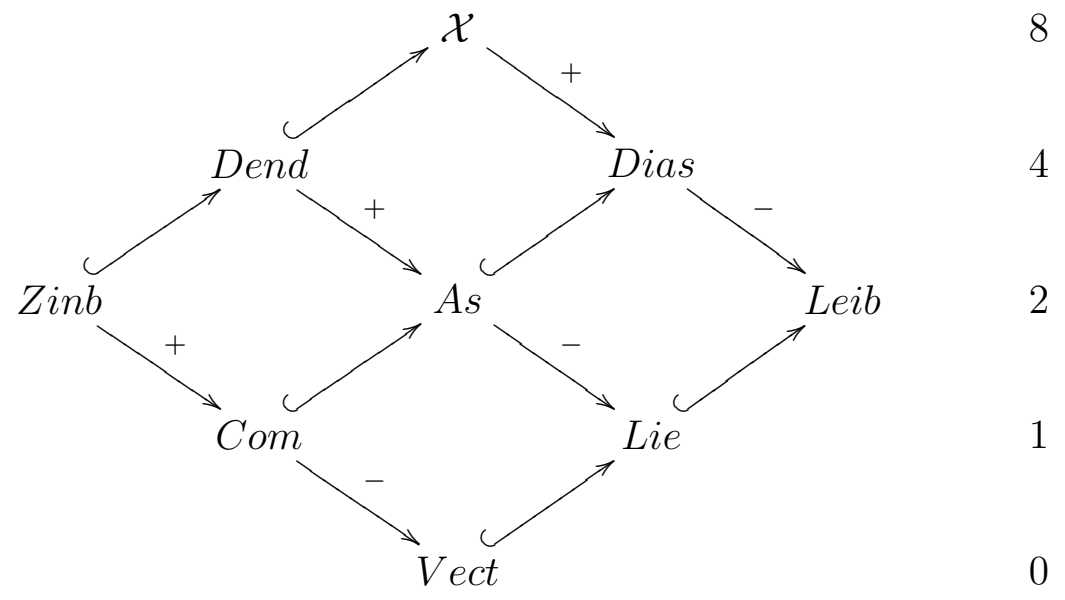

The numbers on the right side of the diagram indicate the dimension of the space of binary operations.

In other words, we would like to find a notion of $\mathcal{X}$-algebra whose operad is binary and quadratic, and satisfies the following properties: 
(1) the space of binary operations is 8-dimensional,

(2) the operad is isomorphic to its dual (for Koszul duality),

(3) a dendriform algebra is an $\mathcal{X}$-algebra satisfying some symmetry,

(4) any $\mathcal{X}$-algebra gives, by some symmetrization of the operations, a diassociative algebra,

(5) the functors deduced from the preceding two items make the upper square of the completed operadic butterfly commutative.

The aim of this paper is to answer this question, and the answer is as follows: there are two solutions $\mathcal{X}^{+}$and $\mathcal{X}^{-}$.

An algebra of type $\mathcal{X}^{ \pm}$has four generating operations denoted by and $16=5 \times 3+1$ relations (we write $(\circ) \bullet$ instead of $(x \circ y) \bullet z)$ :

$$
\begin{array}{ccc}
(\nwarrow) \nwarrow=\nwarrow(\nwarrow)+\nwarrow(\swarrow), & (\swarrow) \nwarrow=\swarrow(\nwarrow), & (\nwarrow) \swarrow+(\swarrow) \swarrow=\swarrow(\swarrow), \\
(\nwarrow) \nwarrow=\nwarrow(\searrow)+\nwarrow(\nearrow), & (\swarrow) \nwarrow=\swarrow(\nearrow), & (\nwarrow) \swarrow+(\swarrow) \swarrow=\swarrow(\searrow), \\
(\nearrow) \nwarrow=\nearrow(\nwarrow)+\nearrow(\swarrow), & \searrow) \nwarrow=\searrow(\nwarrow), & (\nearrow) \swarrow+(\searrow) \swarrow=\searrow(\swarrow), \\
(\nwarrow) \nearrow=\nearrow(\nearrow)+\nearrow(\searrow), & (\swarrow) \nearrow=\searrow(\nearrow), & (\nwarrow) \searrow+(\swarrow) \searrow=\searrow(\searrow), \\
(\nearrow) \nearrow=\nearrow(\nearrow)+\nearrow(\searrow), & (\searrow) \nearrow=\searrow(\nearrow), & (\nearrow) \searrow+(\searrow) \searrow=\searrow(\searrow), \\
(\nearrow) \searrow-(\nwarrow) \searrow= \pm(\nwarrow(\swarrow)-\nwarrow(\searrow)) . & (16 \pm)
\end{array}
$$

A more conceptual way of describing $\mathcal{X}^{ \pm}$is as follows. Given two binary quadratic operads $\mathcal{P}$ and $\mathcal{Q}$ with a preferred basis for the space of generating operations, one can construct a new operad, denoted by $\mathcal{P} \boldsymbol{Q} \mathcal{Q}$, whose set of generating operations is the product of those of $\mathcal{P}$ and $\mathcal{Q}$ and the set of relations is also the product (in a certain sense) of those of $\mathcal{P}$ and $\mathcal{Q}$. This construction has already been used in particular cases in [1], [4] (for $(\mathcal{P}=\mathcal{Q})$ and in [2]. The operad $\mathcal{X}^{ \pm}$is Dend Dias quotiented by the relation (16士). The square product $\mathbf{c}$ constructed here is a variation, in the monoidal category of quadratic regular operads, of Manin's black product [7].

Content. In the first part we recall the theory of Koszul duality for regular operads and introduce the construction $\mathcal{P} \boldsymbol{Q}$. In the second part we introduce the operads Dend and Dias, and compute (Dend $\mathbf{\square}$ Dias)!. In the third part we show that

$$
\mathcal{X}^{ \pm}:=\text {Dend } \boldsymbol{\square} \text { Dias/ relation }(16 \pm)
$$

completes the operadic butterfly.

Convention. All vector spaces are over the field $\mathbb{K}$. The tensor product over $\mathbb{K}$ of the two spaces $V$ and $W$ is denoted by $V \otimes W$.

\section{Product of Operads}

2.1. Regular operads. In this paper we deal with algebras whose structure is defined by generating operations $(x, y) \mapsto x \circ_{i} y$ (with no symmetry) and relations of the form

$$
\sum_{i, j} \alpha_{i j}\left(x \circ_{i} y\right) \circ_{j} z=\sum_{i, j} \beta_{i j} x \circ_{i}\left(y \circ_{j} z\right),
$$


where $\alpha_{i j}$ and $\beta_{i j}$ are scalars. In these relations the variables remain in the same order (this is not the case for Com,Lie, Zinb, Leib). The associated operad, denoted by $\mathcal{P}$, is binary because the generating operations are binary. It is quadratic because the relations involve monomials with two operations. It is regular because the operations have no symmetry and, in the relations the variables stay in the same order. As a consequence of regularity the free $\mathcal{P}$ algebra over the vector space $V$ is of the form

$$
\mathcal{P}(V)=\oplus_{n \geq 1}\left(\mathcal{P}_{n} \otimes \mathbb{K}\left[S_{n}\right]\right) \otimes_{S_{n}} V^{\otimes n}=\oplus_{n \geq 1} \mathcal{P}_{n} \otimes V^{\otimes n}
$$

where $S_{n}$ is the symmetric group. The space $\mathcal{P}_{n}$ is the space of (non-symmetric) $n$-ary operations.

Let us denote by $E=\mathcal{P}_{2}$ the space of (non-symmetric) binary operations. Let $\left\{o_{i} \mid i \in I\right\}$ be a basis of $E$. The space of (non-symmetric) relations $R$ is a subspace of $2 E \otimes E$. The first summand corresponds to the parenthesizing $\left(x \circ_{i} y\right) \circ_{j} z$ and the second summand to the parenthesizing $x \circ_{i}\left(y \circ_{j} z\right)$. It will be helpful to denote by $\left(\circ_{i}\right) \circ_{j}$ and $\circ_{i}\left(\circ_{j}\right)$ the generating elements of $2 E \otimes E$. Hence $R$ is the subspace of $2 E \otimes E$ spanned by the vectors

$$
r=\sum_{i, j} \alpha_{i j}\left(\circ_{i}\right) \circ_{j}-\sum_{i, j} \beta_{i j} \circ_{i}\left(\circ_{j}\right) \in 2 E \otimes E
$$

for each relation $(r)$.

One has $\mathcal{P}_{1}=\mathbb{K}$, since, up to multiplication of scalars, there is only one unary operation: the identity. One has $\mathcal{P}_{2}=E$ and $\mathcal{P}_{3}=(2 E \otimes E) / R$.

If an operad $\mathcal{Q}$ is obtained from an operad $\mathcal{P}$ by enlarging the space of relations with some more relations $(r)$, then the space of $n$-ary operations of $\mathcal{Q}$ is a quotient of the space of $n$-ary operations of $\mathcal{P}$. By abuse of language we will say that $\mathcal{Q}$ is a quotient of $\mathcal{P}$ by $(r)$ and we write $\mathcal{Q}=\mathcal{P} /(r)$.

The Koszul dual of the operad $\mathcal{P}$, denoted by $\mathcal{P}^{!}$, is determined by $E^{*}:=$ $\operatorname{Hom}(E, \mathbb{K})$ and $R^{\perp}$. Since we have equipped $E$ with a basis, we can take the dual basis for $E^{*}$ and identify it with $E$. After this identification $R^{\perp}$ is described as follows: it is the space orthogonal to $R$ for the inner product $\langle-,-\rangle$ given on $E \otimes E \oplus E \otimes E$ by the matrix

$$
\left(\begin{array}{cc}
1 & 0 \\
0 & -1
\end{array}\right)
$$

In other words, one has

$$
\left\{\begin{array}{cl}
\left\langle\left(\circ_{i}\right) \circ_{j},\left(\circ_{i}\right) \circ_{j}\right\rangle=1, & \\
\left\langle\circ_{i}\left(\circ_{j}\right), \circ_{i}\left(\circ_{j}\right)\right\rangle=-1, & \text { in the other cases. }
\end{array}\right.
$$

Since the operations of $\mathcal{P}$ and $\mathcal{P}^{!}$do not satisfy, in general, the same relations, it is necessary, sometimes to distinguish between them. So we adopt the notation $0^{*}$ for the latter. 
Lemma 2.2. Let $K$ be an index set and let $\left(r_{k}\right), k \in K$, be the relations defining $R$. An element

$$
\sum_{i, j} \alpha_{i j}^{\prime}\left(\circ_{i}^{*}\right) \circ_{j}^{*}-\sum_{i, j} \beta_{i j}^{\prime} \circ_{i}^{*}\left(\circ_{j}^{*}\right) \in 2 E^{*} \otimes E^{*}
$$

is in $R^{\perp}$ if and only if one has

$$
\sum_{i, j} \alpha_{i j}^{(k)} \alpha_{i j}^{\prime}=\sum_{i, j} \beta_{i j}^{(k)} \beta_{i j}^{\prime}
$$

for all $k \in K$.

Proof. This is an immediate translation of the definition of orthogonality.

2.3. The square product of operads. Let $\mathcal{P}$ be a binary quadratic regular operad defined by binary operations denoted by $\circ_{i}$, and relations $(r)$ (cf. 2.1). Let $\mathcal{Q}$ be another one with operations $\bullet_{k}$ and relations $\left(r^{\prime}\right)$. We define the operad $\mathcal{P} \boldsymbol{\mathcal { Q }}$ by the operations $\circ_{i} \bullet_{k}$ (product of the two sets of operations), and relations $\left(r, r^{\prime}\right)$ given by

$$
\sum_{i, j, k, l} \alpha_{i j} \alpha_{k l}^{\prime}\left(\circ_{i} \bullet_{k}\right) \circ_{j} \bullet_{l}=\sum_{i, j, k, l} \beta_{i j} \beta_{k l}^{\prime} \circ_{i} \bullet_{k}\left(\circ_{j} \bullet_{l}\right) .
$$

So, if $\mathcal{P}$ is defined by $m$ relations and $\mathcal{Q}$ by $m^{\prime}$ relations, then $\mathcal{P} \mathbf{Q}$ is defined by $\mathrm{mm}^{\prime}$ relations.

It immediately follows that the construction $\mathbf{\square}$ is associative, commutative, and its neutral element is the operad $A s$. Indeed, As has only one operation . satisfying $(\cdot) \cdot=\cdot(\cdot)$.

Proposition 2.4. Let $\mathcal{P}$ and $\mathcal{Q}$ be binary quadratic regular operads. The operad $(\mathcal{P} \square \mathcal{Q}) !$ is a quotient of the operad $\mathcal{P}^{!} \boldsymbol{Q} \mathcal{Q}^{!}$, so there is a natural forgetful functor of categories of algebras:

$$
(\mathcal{P} \square \mathcal{Q})^{!}-\operatorname{alg} \longrightarrow\left(\mathcal{P}^{!} \square \mathcal{Q}^{!}\right)-\text {alg }
$$

Proof. The set of generating operations is the same in both cases, only the space of relations is different. For $(\mathcal{P} \square \mathcal{Q})^{!}$it is $T^{\perp}$, where $T$ is the space generated by the relations $\left(r, r^{\prime}\right)$ (cf. 2.3). For $\mathcal{P}^{!} \mathbf{Q} \mathcal{Q}^{!}$it is the space $S$ generated by the relations $\left(s, s^{\prime}\right)$, where $s$ is orthogonal to all the relations $r$, and $s^{\prime}$ is orthogonal to all the relations $r^{\prime}$.

In order to complete the proof it is sufficient to prove that $S$ is included into $T^{\perp}$. Indeed, the expected functor would then simply be the forgetful functor. The space $S$ is included into $T^{\perp}$ if and only if $\langle S, T\rangle=0$. Let us check this equality for the relations $\left(s, s^{\prime}\right)$ and $\left(r, r^{\prime}\right)$.

We denote by $\alpha, \beta$ the structure constants of $r$ as in 2.1 and by $\gamma, \delta$ the structure constants of $s$ (same thing with a prime for $r^{\prime}$ and $s^{\prime}$ ). By Lemma 2.2 , it suffices to prove the equality

$$
\sum_{i, j, k, l} \alpha_{i j} \alpha_{k l}^{\prime} \gamma_{i j} \gamma_{k l}^{\prime}=\sum_{i, j, k, l} \beta_{i j} \beta_{k l}^{\prime} \delta_{i j} \delta_{k l}^{\prime}
$$

The lefthand summand is equal to 


$$
\left(\sum_{i, j} \alpha_{i j} \gamma_{i j}\right)\left(\sum_{k, l} \alpha_{k l}^{\prime} \gamma_{k l}^{\prime}\right)
$$

and similarly for the righthand side. Since the relations $r$ and $s$ (resp. $r^{\prime}$ and $s^{\prime}$ ) are orthogonal, one has

$$
\sum_{i, j} \alpha_{i j} \gamma_{i j}=\sum_{i, j} \beta_{i j} \delta_{i j}
$$

and similarly with a prime. This proves the equality and so $\langle S, T\rangle=0$.

\section{Dendriform and Diassociative Algebras}

We recall from [5] the definition of dendriform algebra (with a slight change of the notation) and of diassociative algebra.

3.1. Definition. A dendriform algebra $A$ is a vector space equipped with two operations denoted by $\wedge$ and $\vee$ satisfying the following relations:

$$
\left\{\begin{aligned}
(x \wedge y) \wedge z & =x \wedge(y \wedge z)+x \wedge(y \vee z), \\
(x \vee y) \wedge z & =x \vee(y \wedge z), \\
(x \wedge y) \vee z+(x \vee y) \vee z & =x \vee(y \vee z)
\end{aligned}\right.
$$

3.2. Definition. A diassociative algebra (or an associative dialgebra) $A$ is a vector space equipped with two operations denoted by $\dashv$ and $\vdash$ satisfying the following relations:

$$
\left\{\begin{array}{l}
(x \dashv y) \dashv z=x \dashv(y \dashv z), \\
(x \dashv y) \dashv z=x \dashv(y \vdash z), \\
(x \vdash y) \dashv z=x \vdash(y \dashv z), \\
(x \dashv y) \vdash z=x \vdash(y \vdash z), \\
(x \vdash y) \vdash z=x \vdash(y \vdash z) .
\end{array}\right.
$$

It was shown in [5] that the associated operads are dual to each other via the identification $\wedge^{*}=\dashv$ and $\vee^{*}=\vdash$. So we have Dend $d^{!}=$Dias and Dias $s^{!}=$Dend. In fact, the reader can check this immediately from the description of duality for regular operads given in Lemma 2.2.

3.3. The Operad Dend Dias and its dual. Consider the operad Dend Dias. We denote its set of generating operations by

$$
\nwarrow:=(\wedge, \dashv), \quad \nearrow:=(\wedge, \vdash), \searrow:=(\vee, \vdash), \swarrow:=(\vee, \dashv) .
$$

Since Dend has 3 relations and since Dias has 5 relations, Dend Dias has 15 relations which read as follows:

$$
\begin{array}{lll}
(\nwarrow) \nwarrow=\nwarrow(\nwarrow)+\nwarrow(\swarrow), & (\swarrow) \nwarrow=\swarrow(\nwarrow), & (\nwarrow) \swarrow+(\swarrow) \swarrow=\swarrow(\swarrow), \\
(\nwarrow) \nwarrow=\nwarrow(\searrow)+\nwarrow(\nearrow), & (\swarrow) \nwarrow=\swarrow(\nearrow), & (\nwarrow) \swarrow+(\swarrow) \swarrow=\swarrow(\searrow), \\
(\nearrow) \nwarrow=\nearrow(\nwarrow)+\nearrow(\swarrow), & (\searrow) \nwarrow=\searrow(\nwarrow), & (\nearrow) \swarrow+(\searrow) \swarrow=\searrow(\swarrow), \\
(\nwarrow) \nearrow=\nearrow(\nearrow)+\nearrow(\searrow), & (\swarrow) \nearrow=\searrow(\nearrow), & (\nwarrow) \searrow+(\swarrow) \searrow=\searrow(\searrow), \\
(\nearrow) \nearrow=\nearrow(\nearrow)+\nearrow(\searrow), & (\searrow) \nearrow=\searrow(\nearrow), & (\nearrow) \searrow+(\searrow) \searrow=\searrow(\searrow) .
\end{array}
$$

The tableau 
Proposition 3.4. The operad of (Dend Dias)!-algebras is isomorphic to the quotient of the operad Dend Dias by the following two relations

$$
\left\{\begin{array}{l}
(\nearrow) \searrow-(\nwarrow) \searrow=0, \\
0=\nwarrow(\swarrow)-\nwarrow(\searrow) .
\end{array}\right.
$$

Proof. The 15 relations of Dend $\mathbf{\square}$ Dias are linearly independent, hence the space of relations is of dimension 15. Its orthogonal space is of dimension $2 \times$ $4^{2}-15=17$. Since Dend ${ }^{!}=$Dias and Dias ${ }^{!}=$Dend, one has Dend ${ }^{!}$Dias $^{!}=$ Dias Dend $\cong$ Dend Dias. By Proposition 2.4 (Dend Dias)! has, at least, the 15 relations of Dend Dias (up to isomorphism) as relations plus two more (linearly independent). Let us show that the two extra operations are the ones indicated.

We first make explicit the isomorphism:

$$
\begin{aligned}
& \nwarrow^{*}=(\wedge, \dashv)^{*}=\left(\wedge^{*}, \dashv^{*}\right)=(\dashv, \wedge) \cong \nwarrow, \\
& \nearrow^{*}=(\wedge, \vdash)^{*}=\left(\wedge^{*}, \vdash^{*}\right)=(\dashv, \vee) \cong \swarrow, \\
& \searrow^{*}=(\vee, \vdash)^{*}=\left(\vee^{*}, \vdash^{*}\right)=(\vdash, \vee) \cong \searrow, \\
& \swarrow^{*}=(\vee, \dashv)^{*}=\left(\vee^{*}, \dashv^{*}\right)=(\vdash, \wedge) \cong \nearrow .
\end{aligned}
$$

So, in order to write down the 15 relations of (Dend $\mathbf{\square}$ Dias)! with this basis of generating operations we have to exchange the operations $\nearrow$ and $\measuredangle$ in the tableau of the 15 relations. Let us call it the dual tableau. Under this isomorphism the two extra relations read as follows

$$
\left\{\begin{array}{l}
(\swarrow) \searrow-(\nwarrow) \searrow=0 \\
0=\nwarrow(\nearrow)-\nwarrow(\searrow)
\end{array}\right.
$$

Let us check that these two relations are orthogonal with the 15 relations of Dend Dias. In 28 cases the verification is immediate because the involved operations are all different. The remaining two cases are

$$
\begin{aligned}
& \langle(\swarrow) \searrow-(\nwarrow) \searrow,(\nwarrow) \searrow+(\swarrow) \searrow-\searrow(\searrow)\rangle=+1-1=0, \\
& \langle\nwarrow(\nearrow)-\nwarrow(\searrow),-(\nwarrow) \nwarrow+\nwarrow(\searrow)+\nwarrow(\nearrow)\rangle=-1+1=0 .
\end{aligned}
$$

It is straightforward to check that these 17 relations are linearly independent, therefore we have a complete presentation of (Dend Dias)! Under the inverse isomorphism of the one described above we get the expected result.

\section{The Missing Operad}

In this section we complete the operadic butterfly by constructing the op$\operatorname{erad} \mathcal{X}$.

Theorem 4.1. Let $\mathcal{X}^{+}$(resp. $\mathcal{X}^{-}$) be the operad Dend $\mathbf{\square}$ Dias quotiented by the relation $(16+)$ (resp. $(16-))$ :

$$
\begin{array}{ll}
(\nearrow) \searrow-(\nwarrow) \searrow=+\nwarrow(\swarrow)-\nwarrow(\searrow), & (16+) \\
(\nearrow) \searrow-(\nwarrow) \searrow=-\nwarrow(\swarrow)+\nwarrow(\searrow) . & (16-)
\end{array}
$$

The operad $\mathcal{X}^{ \pm}$completes the operadic butterfly, that is, it satisfies the following properties: 
(1) the space of binary operations is 8-dimensional,

(2) the operad is isomorphic to its dual (for Koszul duality),

(3) a dendriform algebra is an $\mathcal{X}$-algebra satisfying some symmetry,

(4) any $\mathcal{X}$-algebra gives, by some symmetrization of the operations, a diassociative algebra,

(5) the functors deduced from the preceding two items make the upper square of the completed operadic butterfly commutative.

Proof. Let us put $r:=(\nearrow) \searrow-(\nwarrow) \searrow$ and $s:=\nwarrow(\swarrow)-\nwarrow(\searrow)$. We are looking for scalars $\alpha$ and $\beta$ such that the operad Dend Dias/ $\mathbf{\square}+\beta s$ is self-dual. The dual of $r$ (resp. $s$ ) is $r^{*}=(\swarrow) \searrow-(\nwarrow) \searrow\left(\right.$ resp. $s^{*}=\nwarrow$ $(\nearrow)-\nwarrow(\searrow))$. To get the self-duality of the quotient operad we need to have $-\alpha^{2}+\beta^{2}=0$. Hence $\alpha$ and $\beta$ have to be equal up to sign. So the sixteenth relation is either $r=s$ called $(16+)$ or $r=-s$, called (16-). It can be checked immediately that in both cases the $16(=3 \times 5+1))$ relations are linearly independent. Hence we have proved (1) and (2). It turns out that both solutions satisfy also the other requirements as we prove now.

(3) Let $(A, \wedge, \vee)$ be a dendriform algebra. Define $\nwarrow:=\wedge=: \nearrow$ and $\swarrow:=$ $\vee=: \searrow$. Let us prove that $(A, \nwarrow, \nearrow, \swarrow, \searrow)$ is an $\mathcal{X}^{ \pm}$-algebra. The first fifteenth relations are fulfilled since relation $(n, a)$ is a consequence of relation $(a)$ for $n=1, \ldots, 5$ and $a=i, i i, i i i$. The relation $(16 \pm)$ is also fulfilled since both sides of the equality are 0 : the left side because $\nwarrow=\nearrow$ and the right side because $\swarrow=\searrow$.

(4) Let $(A, \nwarrow, \nearrow, \swarrow, \searrow)$ be an $\mathcal{X}^{ \pm}$-algebra. Define

$$
x \dashv y:=x \nwarrow y+x \swarrow y \text { and } \quad x \vdash y:=x \nearrow y+x \searrow y \text {. }
$$

Adding the relations $(n, i),(n, i i)$ and $(n, i i i)$ shows that the relation $(n)$ is fulfilled for $n=1, \ldots, 5$.

(5) Starting with a dendriform algebra $(A, \wedge, \vee)$, we get a diassociative algebra $(A, \dashv, \vdash)$ such that

$$
x \dashv y=x \nwarrow y+x \swarrow y=x \wedge y+x \vee y=x \nearrow y+x \searrow y=x \vdash y .
$$

Therefore the composite Dend $\hookrightarrow \mathcal{X}^{ \pm} \stackrel{+}{\rightarrow}$ Dias is equal to the composite Dend $\stackrel{+}{\rightarrow}$ As $\hookrightarrow$ Dias as expected.

Remark. In fact, our proof shows that these two solutions are the only quotients of Dend Dias which satisfy all the required properties.

4.2. Question. The operads Com, Lie, As, Zinb, Leib, Dend, Dias are Koszul operads, i.e. the associated Koszul complex is acyclic (cf. [3], [6], [5]). Is also $\mathcal{X}^{+}\left(\right.$resp. $\left.\mathcal{X}^{-}\right)$a Koszul operad?

Since the operad $\mathcal{X}\left(=\mathcal{X}^{+}\right.$or $\left.\mathcal{X}^{-}\right)$is regular and self-dual, the criterion to ensure Koszul duality takes the following form. Let $\mathcal{X}_{n}$ be the homogeneous part of degree $n$ in the free $\mathcal{X}$-algebra on one generator. Then for each integer $k \geq 1$ there is a finite chain complex 


$$
\begin{aligned}
0 \rightarrow \mathcal{X}_{k} \otimes & \mathcal{X}_{1} \otimes \cdots \otimes \mathcal{X}_{1} \rightarrow \cdots \\
& \rightarrow \bigoplus_{m_{1}+\cdots+m_{n}=k} \mathcal{X}_{n} \otimes \mathcal{X}_{m_{1}} \otimes \cdots \otimes \mathcal{X}_{m_{n}} \rightarrow \cdots \\
& \cdots \rightarrow \mathcal{X}_{1} \otimes \mathcal{X}_{k} \rightarrow 0
\end{aligned}
$$

The acyclicity of these complexes for $k>1$ implies Koszulity. It implies that $\mathcal{X}_{n}$ is of dimension $4^{n-1}$.

Added in proofs. The Koszulity of the operad Dend Dias has been recently proved in B. VALLETTE, Manin products, Koszul duality, Loday algebras and Deligne conjecture (2006), ArXiv math.QA/0609002.

\section{REFERENCES}

1. M. Aguiar and J.-L. Loday, Quadri-algebras. J. Pure Appl. Algebra 191(2004), No. $3,205-221$.

2. K. Ebrahimi-Fard and L. Guo, On products and duality of binary, quadratic, regular operads. J. Pure Appl. Algebra 200(2005), No. 3, 293-317.

3. V. Ginzburg and M. Kapranov, Koszul duality for operads. Duke Math. J. 76(1994), No. 1, 203-272.

4. Ph. Leroux, Ennea-algebras. J. Algebra 281(2004), No. 1, 287-302.

5. J.-L. Loday, Dialgebras. Dialgebras and related operads, 7-66, Lecture Notes in Math., 1763, Springer, Berlin, 2001.

6. J.-L. Loday and T. Pirashvili, Universal enveloping algebras of Leibniz algebras and (co)homology. Math. Ann. 296(1993), No. 1, 139-158.

7. Yu. I. Manin, Quantum groups and noncommutative geometry. Université de Montréal, Centre de Recherches Mathématiques, Montreal, QC, 1988.

(Received 5.07.2006)

Author's address:

Institut de Recherche Mathématique Avancée

CNRS et Université Louis Pasteur

7 rue R. Descartes

67084 Strasbourg Cedex, France

E-mail: loday@math.u-strasbg.fr

$U R L$ : www-irma.u-strasbg.fr/ loday/ 